\title{
Mapping organic functional groups at nanosurfaces using colloidal gold conjugation
}

Nicolas Debons, ${ }^{1}$ Dounia Dems, ${ }^{1}$ Thibaud Coradin, ${ }^{1}$ Carole Aimé ${ }^{1,2 *}$

${ }^{1}$ Sorbonne Université, CNRS, Laboratoire de Chimie de la Matière Condensée de Paris (LCMCP), Paris, F-75005, France

${ }^{2}$ Ecole Normale Supérieure, CNRS-ENS-SU UMR 8640, 24 rue Lhomond, Paris, 75005, France

* Corresponding author: carole.aime@ens.psl.eu 


\section{ABSTRACT}

The functionalization of the surface of nanomaterials is a key approach to improve their stability, tune their reactivity and confer them specific properties. However, the mapping of functional groups at the nanometer scale remains difficult to achieve. This concerns not only the chemical nature of these groups, but also their spatial distribution. It is particularly challenging when it comes to organic groups and non-planar objects such as nanoparticles and necessitates the development of strategies beyond the techniques (i.e. chemical analysis and imaging). Here we report such a strategy for mapping amine groups on the surface of silica particles using chemicallymodified gold colloids, which are used as tags to specifically and spatially identify these organic groups under electron microscopy. A complete understanding of the correlation between the spatial distribution of gold colloids and the chemical state of the silica particle surface, as obtained by Xray photoelectron spectroscopy, is presented. The range of suitability and reliability of this strategy for mapping organic groups at nanomaterial interfaces is assessed, and its implications for biofunctional nano-objects where interdistance and clustering of signaling biomolecules are of paramount importance are discussed. 


\section{INTRODUCTION}

In nanomedicine and nanobiotechnology, biomolecules such as nucleic acids, ${ }^{1,2}$ peptides and proteins $^{3,4}$ can be conjugated at the surface of nanomaterials, so as to tune their interaction with the living environment. Beside the chemical nature of the biological ligand, its spatial distribution and clustering is key to drive a given cell behavior. ${ }^{5}$ However, achieving such a controlled positioning of functions on nanosurfaces remains highly challenging. This requires the formation of chemical clusters that result from the inhomogeneous distribution of functional groups, and that in turns provides access to distinct surface chemistries, hence functionalities in and out clusters. The need for clustering and bifunctionality in biomaterial engineering makes patchy particles very attractive as they provide at least one chemically-defined domain or patch. In the last decades, many strategies have been developed to synthesize patchy particles, including Janus nanoparticles, by vapor deposition onto a nanoparticle monolayer (glancing-angle vapor deposition, ${ }^{6}$ lithography ${ }^{7}$ ), or in a liquid environment. In this case, several approaches have been described, such as templateassisted fabrication, where only one patch is created, ${ }^{8,9}$ Pickering emulsion, ${ }^{10,11}$ and lithography. ${ }^{7}$ Moreover, direct self-assembly of particles through evaporation-driven colloidal assembly was described. ${ }^{12,13}$ Liquid streams in capillaries have also been used to create multiphasic particles. ${ }^{14,15}$ Other alternatives include seeded emulsion polymerization at the surface of silica nanoparticles for the formation of polystyrene, gold or silica nodules. ${ }^{16-18}$ In addition, molecular approaches have been developed to create localized patches using anisotropic particles that exhibit spatially-defined reactivities, ${ }^{19-21}$ or based on the segregation of functional ligands during particle synthesis. ${ }^{22}$

Characterization of chemically heterogeneous nanosurfaces remains highly challenging per se. ${ }^{23,24}$ From the chemical viewpoint, changes in surface chemistry can be monitored from zeta potential measurements or charge titration, UV-vis or fluorescence spectroscopy for the grafting of 
specific moieties, ${ }^{25}$ or by NMR, as reported for the statistical determination of two types of grafted ligands at silica nanosurface. ${ }^{26}$ Alternatively, X-ray photoelectron spectroscopy (XPS) is used to analyze chemical elements together with their environment, which can be discriminated with a remarkable accuracy. ${ }^{27}$ However, these techniques lack structural information regarding the spatial distribution of functional groups on the surface at the nanoscale. Such a correlation can be implemented thanks to energy-dispersive X-ray (EDX) or electron energy loss spectroscopy (EELS) combined to electron microscopy (EM). ${ }^{28,29}$ This provides a rapid and practical tool for nanoanalysis of both morphology and elemental composition. However, EDX and EELS have a low sensitivity towards light elements, typically carbon and nitrogen. Finally, atomic force microscopy (AFM) has been shown to be efficient for probing the formation of organosilane domains on silica surface, ${ }^{30}$ but facing difficulties when characterizing 3D nanoparticles. ${ }^{31}$

Overall, mapping the position of organic functional groups on nanoparticle surface is crucial in nanotechnology for improving the functionality of composite biomaterials. We have recently reported that the simultaneous display of two signaling peptides on silica nanoparticles (SiNPs) significantly enhances cell response compared to the presentation of these two peptides independently, i.e. on two different SiNP populations. ${ }^{32}$ Despite these key results, it remains difficult to precisely determine the inter-peptide distance at the surface of the particles, a key to achieve in order to faithfully reproduce the organization of living systems. To address this challenge we propose here to use chemically-modified colloidal gold as tag to reveal the position of amine groups on nanosurfaces taking advantage of the robustness and specificity of peptide-like coupling. Carboxylate-modified gold colloids (AuNP) can be specifically conjugated to SiNPs modified with amine groups from (3-Aminopropyl)triethoxysilane (APTES). The spatial mapping of AuNPs (by EM) combined with zetametry and XPS allows to fully address the chemical state of the SiNP surface with varying amine density. This ultimately provides a new methodology to 
correlate chemical grafting with mapping of functional groups. Beyond its interest for the engineering of composite biomaterials, for which the spatial distribution of biological ligands is particularly crucial, this methodology will find many echoes and developments in all fields of nanotechnologies, where densities and nanoscale inter-distances greatly impact functions.

\section{METHODS}

Synthesis of Bare Silica Nanoparticles (SiNPs). According to the Stöber procedure, 21 mL tetraethyl orthosilicate (TEOS 98\%, Sigma Aldrich) was added dropwise to a solution containing $32 \mathrm{~mL}$ ultrapure water, $600 \mathrm{~mL}$ absolute ethanol (VWR, GPR RectaPur) and $45 \mathrm{~mL}$ ammonium hydroxide solution (25\%, Carlo Erba). The solution was left under stirring overnight at RT. SiNPs were washed with ethanol through an ultrasonic redispersion-centrifugation process (10 $000 \mathrm{rpm}$ for $5 \mathrm{~min}$ ) and dried overnight at $30^{\circ} \mathrm{C}$ under vacuum. Particle size was $417 \pm 26 \mathrm{~nm}$, as determined by transmission electron microscopy.

Synthesis of Amine-Grafted Silica Nanoparticles (SiNP-NH2). In a typical experiment, SiNPs $(15 \mathrm{mg}, 250 \mu \mathrm{mol})$ were dispersed in a mixture of $1.5 \mathrm{~mL}$ ethanol and $3.3 \mu \mathrm{L}$ ammonium hydroxide solution before addition of (3-Aminopropyl)triethoxysilane (APTES 99\%, Aldrich). APTES volume was varied between $0.75 \mathrm{~nL}(0.0032 \mu \mathrm{mol})$ and $15 \mu \mathrm{L}(64 \mu \mathrm{mol})$. The mixture was stirred for 2 hours at RT. Then, the reaction mixture was heated to $80^{\circ} \mathrm{C}$ and the volume was reduced to approximately two-thirds by distillation of ethanol and ammonia at ambient pressure. The mixture was left to cool down to RT and subsequently washed twice with ethanol through an ultrasonic redispersion-centrifugation process (10 $000 \mathrm{rpm}$ for $5 \mathrm{~min}$ ) and dried overnight at $30^{\circ} \mathrm{C}$ under vacuum.

Synthesis of Carboxylate-Modified Gold Nanoparticles (AuNP). Based on the protocol of Chen et al. ${ }^{33} 0.5 \mathrm{mmol}$ of hydrogen tetrachloroaurate tetrahydrate $\left(\mathrm{HAuCl}_{4}, 4 \mathrm{H}_{2} \mathrm{O}\right.$, Wako Pure 
Chemical Co, 99\%) dissolved as a $4 \%$ (w/v) aqueous solution was mixed with 0.25 mmol of mercaptosuccinic acid (Sigma Aldrich 97\%) in $100 \mathrm{~mL}$ methanol to give a transparent solution. A freshly-prepared $0.2 \mathrm{M}$ sodium borohydride aqueous solution $(25 \mathrm{~mL})$ was then added at a rate of $5 \mathrm{~mL}$ per min under vigorous stirring. The solution immediately turned dark-brown but remained transparent until approximately $13 \mathrm{~mL}$ of the reductant were added. After further stirring for $1 \mathrm{~h}$, the solvent was removed by decantation after centrifugation at $10000 \mathrm{rpm}$ for $5 \mathrm{~min}$. The precipitate was washed twice with a $20 \%(\mathrm{v} / \mathrm{v})$ water/methanol solution through an ultrasonic redispersion-centrifugation process to remove impurities. This process was further repeated with methanol and ethanol and particles were dried under vacuum overnight at $40^{\circ} \mathrm{C}$.

Gold Nanoparticle Conjugation. 1.36 mg AuNP (200 eq) and 2.1 mg 1[Bis(dimethylamino)methylene]-1H-1,2,3-triazolo[4,5-b]pyridinium 3-oxid hexafluorophosphate (Hexafluorophosphate Azabenzotriazole Tetramethyl Uronium, HATU) (400 eq) were dispersed in $300 \mu \mathrm{L}$ DMF and $0.95 \mu \mathrm{L}$ N,N-Diisopropylethylamine (DIEA) (400 eq) was added. The solution rapidly turned yellow. The mixture underwent a vortex redispersion-centrifugation process for 15 $\mathrm{s}$, and was sonicated for 5 minutes before adding $0.8 \mathrm{mg} \mathrm{SiNP}-\mathrm{NH}_{2}(1 \mathrm{eq})$. Vortex was used once again and the mixture was sonicated for 3 hours. Every hour during that time the solution underwent a vortex redispersion-centrifugation process (7 $500 \mathrm{rpm}$ for $15 \mathrm{~s}$ ). The mixture was washed with DMF and water through an ultrasonic redispersion-centrifugation process (10 $000 \mathrm{rpm}$ for $5 \mathrm{~min}$ ).

Dynamic Light Scattering (DLS) and Zeta Potential Measurements. DLS and zetapotential measurements were performed on a Malvern Zetasizer Nano spectrometer. The autocorrelation functions were recorded at a scattering angle $\Theta=90^{\circ}$ and analyzed by the nonnegatively constrained least squares model (NNLS-Multiple Pass) for the determination of the particle hydrodynamic diameter. DLS measurements were made at ca. $0.05 \mathrm{~g} . \mathrm{L}^{-1}$ at RT in water 
for AuNPs. Zeta potential measurements were performed at 0.05 g. $\mathrm{L}^{-1}$ in DTS1060C cells at $25^{\circ} \mathrm{C}$ in $100 \mathrm{mM} \mathrm{KCl}$ buffer at different $\mathrm{pHs}$ adjusted by addition of $\mathrm{NaOH}$ and $\mathrm{HCl}$.

X-ray Photoelectron Spectroscopy (XPS). XPS analyses were performed using an Omicron Argus X-ray photoelectron spectrometer, equipped with a monochromated $\mathrm{AlK}_{\alpha}$ radiation source $(\mathrm{h} v=1486.6 \mathrm{eV})$ and a $280 \mathrm{~W}$ electron beam power. The emission of photoelectrons from the sample was analyzed at a takeoff angle of $45^{\circ}$ under ultra-high vacuum conditions $\left(\leq 10^{-9}\right.$ mBar). Spectra were carried out with a $100 \mathrm{eV}$ pass energy for the survey scan and $20 \mathrm{eV}$ pass energy for the $\mathrm{C} 1 \mathrm{~s}, \mathrm{O} 1 \mathrm{~s}, \mathrm{~N}$ 1s, Si 2p regions. Binding energies were calibrated against the $\mathrm{C} 1 \mathrm{~s}$ (C-C) binding energy at $284.8 \mathrm{eV}$ and element peak intensities were corrected by Scofield factors. The peak areas were determined after substraction of a Shirley background. The spectra were fitted using Casa XPS v.2.3.15 software (Casa Software Ltd, U.K.). Inelastic electron mean free paths were calculated from the TPP-2M formula thanks to the software QUASES-IMFP-TPP2M Ver. 3.0 .

Transmission Electron Microscopy (TEM). A drop of sample in aqueous solution was deposited on carbon-coated copper grids (300 mesh). After 3 minutes, the excess liquid was blotted with filter paper. TEM was performed using a Tecnai spirit G2 electron microscope operating at $120 \mathrm{kV}$ and the images were recorded on a Gatan Orius CCD camera. Determination of AuNP diameter was made by analyzing the size of 100 AuNPs thanks to ImageJ software. Determination of AuNP density on particle surface was achieved by measuring the number of AuNPs on a crown of 20 nanometers (4 pictures at high magnification) for 10 SiNPs for each condition. The limit of $20 \mathrm{~nm}$ has been defined in order to make a compromise between the highest possible magnification - thus with an improved discrimination of AuNPs - and a representative area relative to the particle surface $(20 \mathrm{~nm}$ represents less than $5 \%$ of SiNP diameter). 


\section{RESULTS}

Synthesis and Characterization of Amine-Modified Silica Nanoparticles. Surface modification of $417 \pm 26 \mathrm{~nm}$ SiNPs with (3-Aminopropyl)triethoxysilane (APTES) was performed in ethanol in the presence of ammonium hydroxide. The reaction was performed at fixed silica content and the amount of APTES was varied from 0.0125 to $250 \mathrm{mmol}$ per mol of $\mathrm{SiO}_{2}$. The different samples were numbered from $\mathbf{1}$ (no APTES) to $\mathbf{1 0}\left(250 \mathrm{mmol}\right.$ APTES/mol $\left.\mathrm{SiO}_{2}\right)$, and the corresponding APTES:silanol molar ratios were calculated, assuming a silanol density of $c a .4 .5$ SiOH.nm ${ }^{2}$ on SiNP surface, i.e. $2.5 \mathrm{mmol} \mathrm{SiOH/mol} \mathrm{SiO} 2$, ranging from 0.005:1 (2) to 100:1 (10) (Table 1).

Table 1. APTES:SiOH molar ratio, N/Si Atomic Ratio, N1s Binding Energy, $\mathrm{NH}_{2} / \mathrm{NH}_{3}{ }^{+}$Peaks Intensity Ratio and Estimated Amine Density

$\begin{array}{cccccc}\# & \begin{array}{c}\text { Initial } \\ \text { APTES:SiOH } \\ \text { molar ratio }\end{array} & \begin{array}{c}\mathrm{N} / \mathrm{Si} \\ \text { atomic ratio }^{a}\end{array} & \begin{array}{c}\mathrm{N} 1 \mathrm{~s} \text { Binding energy } \\ (\mathrm{eV})\end{array} & \begin{array}{c}\mathrm{NH}_{2} / \mathrm{NH}_{3}{ }^{+} \\ \text {peaks } \\ \text { intensity ratio }\end{array} & \begin{array}{c}\text { Estimated }^{a} \\ \text { amines.nm }^{2}\end{array} \\ \mathbf{1} & 0 & 0.004 & {[400.5 ; 402.0]} & 1.5 & <1 \\ \mathbf{2} & 0.005 & 0.005 & {[399.9 ; 402.0]} & 1.9 & <1 \\ \mathbf{3} & 0.0075 & 0.004 & {[399.7 ; 401.5]} & 1.8 & <1 \\ \mathbf{4} & 0.01 & 0.009 & {[399.9 ; 401.8]} & 2.6 & 1 \\ \mathbf{5} & 0.025 & 0.012 & {[399.8 ; 402.0]} & 2.5 & 2 \\ \mathbf{6} & 0.05 & 0.014 & {[399.6 ; 401.9]} & 2.9 & 2 \\ \mathbf{7} & 0.1 & 0.040 & {[399.7 ; 401.6]} & 2.5 & 5 \\ \mathbf{8} & 1 & 0.053 & {[398.6 ; 400.7]} & 2.6 & 7 \\ \mathbf{9} & 10 & 0.075 & {[399.1 ; 401.0]} & 1.2 & 9 \\ \mathbf{1 0} & 100 & 0.065 & {[399.2 ; 401.1]} & 1.3 & 8 \\ & & & & & \end{array}$

${ }^{a}$ From XPS measurements 

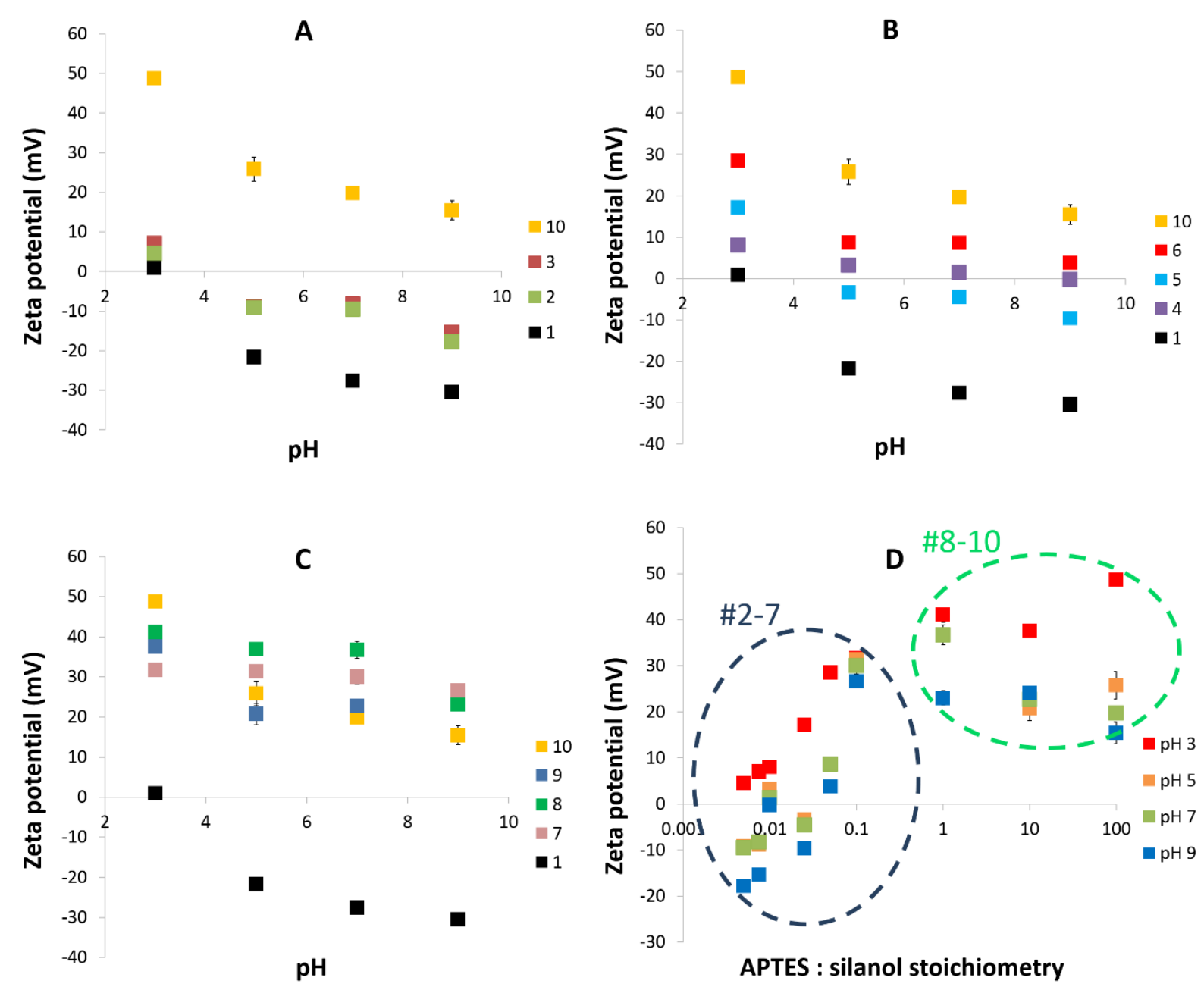

Figure 1. Zeta potential as a function of $\mathrm{pH}$ (in $100 \mathrm{mM} \mathrm{KCl}$ ) for samples (A) 2-3, (B) 4-6, and (C) 7-9 with bare SiNPs (\#1) and sample \#10 as references. (D) Zeta potential as a function of APTES:silanol ratio.

Zeta potentials were first measured for all samples as a function of $\mathrm{pH}$ and the results presented in three graphs in sake of clarity, where zeta potentials of sample $\mathbf{1}$ and $\mathbf{1 0}$ are plotted as references (Figure 1). Samples $\mathbf{2}$ and $\mathbf{3}$ had close zeta values, that were slightly positive at pH 3 $(c a .+5 \mathrm{mV})$ and decreased with increasing $\mathrm{pH}$ down to $c a .-15 \mathrm{mV}$ (Figure 1A). These values were significantly higher than for $\mathbf{1}$ over the whole range of $\mathrm{pHs}$, which exhibited a zeta potential of $-30 \mathrm{mV}$ at $\mathrm{pH}$ 9. Concerning samples 4-6, zeta potentials stood between those of $\mathbf{1}$ and $\mathbf{1 0}$, increasing with aminosilane content at $\mathrm{pH} 3$ and being higher for sample $\mathbf{6}$ than for samples $\mathbf{4}$ and 
5 over the whole range of pHs (Figure 1B). However, zeta values for 5, which evolve in a nonmonotonous way, were smaller than for 4 in the 5-9 pH range. For high initial APTES amount, samples 7-10 behave very similarly, zeta potentials being comparable and positive over the whole $\mathrm{pH}$ range (Fig. 1-C). However, except at $\mathrm{pH} 3$, the $\zeta$ values did not systematically increase with the initial aminosilane content and, at $\mathrm{pH} 9$, the lowest $\zeta$ value was measured for $\mathbf{1 0}$. These evolutions are better evidenced on Figure 1D that gathers the $\zeta$ values of all samples at $\mathrm{pH} 3,5,7$ and 9. All samples have a positive zeta value at $\mathrm{pH}$, that increases as the initial APTES:silanol ratio increases. At higher $\mathrm{pHs}$, there is a general trend towards the increase of the zeta potential from samples 2 to 7 and a plateau seems to be reached from sample 7.

XPS was then used to quantify the amount of nitrogen on the nanoparticle surface and gain insights on amine groups charge and environment. For the bare SiNPs (1), Si (41.7 \%) and O (53.7 $\%)$ are clearly identified, together with $\mathrm{C}(4.5 \%)$ and $\mathrm{S}(<0.1 \%)$ that is attributed to contamination (see Figure 2.1-full and Table S1 for the corresponding atomic percentages). For sample 1, nitrogen is also present, although in a very small amount $(0.16 \mathrm{At} \%)$. For sample 10, $\mathrm{C}$ and $\mathrm{N}$ contents have significantly increased $(15.6 \%$ and $2.39 \%$, respectively), while O and Si contents have decreased (44.7\% and $37.4 \%$, respectively). In agreement with APTES grafting, the C1s peak was narrower for $\mathbf{1 0}$ compared to $\mathbf{1}$ and shifted towards lower binding energy signing for aliphatic carbons of the APTES propyl chain (see Figure S1-S4 for the detailed XPS data). Accordingly, a narrowing and low-energy shift is observed for the $\mathrm{Si} 2 \mathrm{p}$ peak that reflects the presence of the silicon atom of the aminosilane groups that is in a lower oxidation state than in silanol or siloxane groups. 

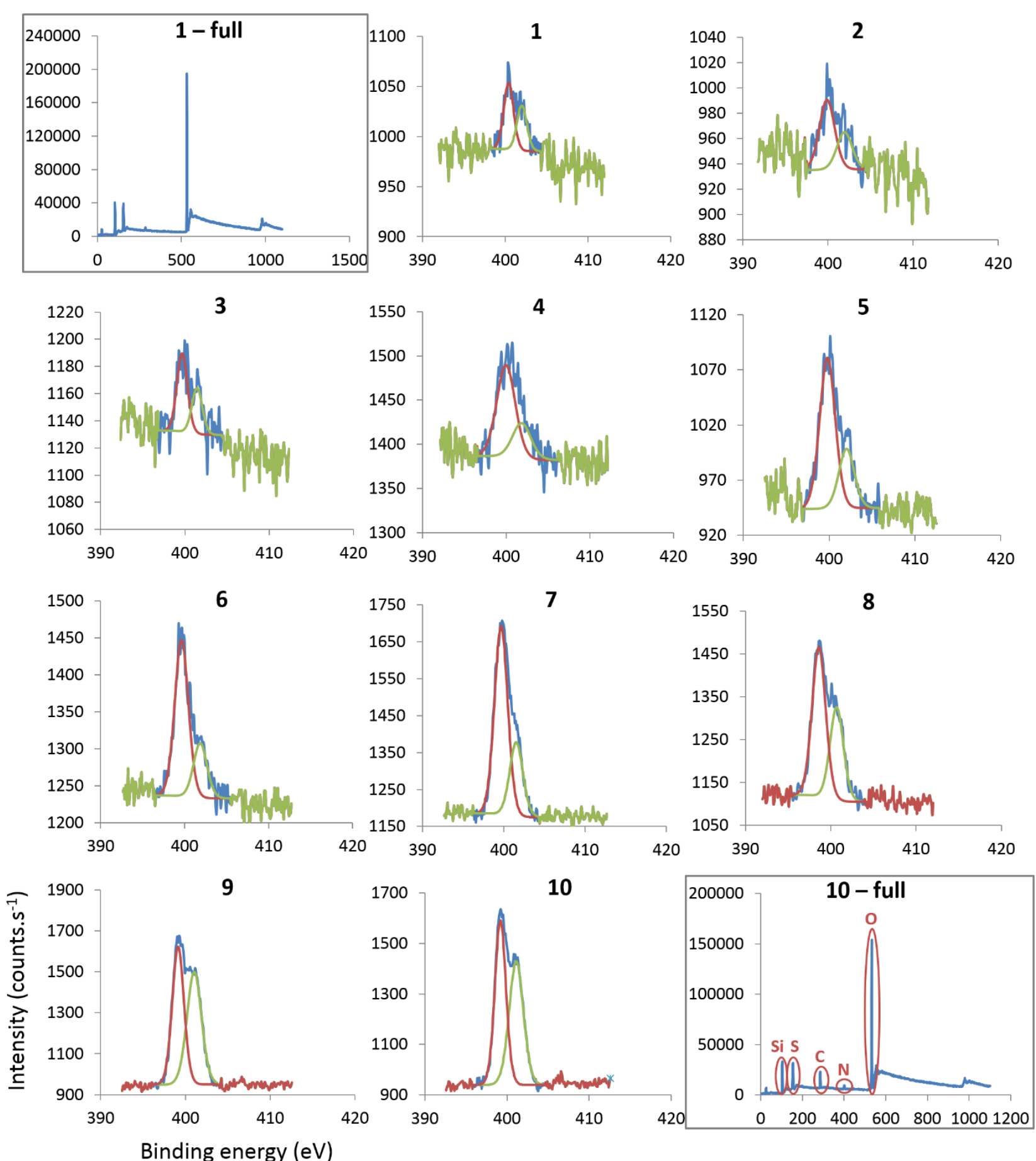

Figure 2. XPS spectra of the amine-modified samples: full spectra of sample $\mathbf{1}$ and $\mathbf{1 0}$ and N1s spectra for samples 110 (blue line: experimental data; red and green lines: calculated peaks from fitting procedure). For clarity the axes have been labeled for a single XPS spectrum; intensity (counts.s-1) on y axis, and binding energy (eV) on x axis. 
Table 1 shows the calculated N/Si atomic ratio for all samples. From these data, the presence of grafted APTES is ascertained from sample 4, where the N/Si ratio is significantly higher than for the bare particles, and its amount increased with initial silane content until sample 9. Two peaks can be identified at the N1s threshold (see the red and green lines in Figure 2), whose binding energies tend to decrease with increasing APTES concentration (Table 1). The peak of lower energy that is attributed to neutral amine groups, was always more intense than the one at higher energy, assigned to ammonium groups. However, the intensity ratio varied nonmonotonously with aminosilane initial content, with a global increase for the first six samples and decreasing afterwards (Table 1).

Gold Nanoparticle Conjugation and Mapping. AuNPs were selected as electron-dense tags to be imaged by TEM to identify amines after specific conjugation. In terms of dimensions, a balance had to be found between sizes small enough for AuNP to interact with a minimum number of grafted moieties, i.e. optimize the resolution of the detection, and large enough for TEM imaging. From these considerations, we prepared carboxylate-modified AuNPs $(5.0 \pm 0.7 \mathrm{~nm}$ in diameter) based on the reduction of hydrogen tetrachloroaurate(III) by sodium borohydride in methanol using mercaptosuccinic acid (MSA) as the stabilizing thiol ligand (see Supporting Information, Figure S5 and Table S2) ${ }^{33}$ MSA-stabilized AuNPs were conjugated to APTESmodified SiNPs via a peptide coupling reaction involving HATU and DIEA. As a matter of fact, preliminary attempts using $\mathrm{HOBt} / \mathrm{HBTU}$ coupling showed poor reproducibility of the resulting AuNP coating (see Supporting Information and Figure S6). The efficient HATU/DIEA coupling system was selected to optimize colloid conjugation based on peptide coupling, as it was reported that (1) the transitional activated ester arising from its reaction with the carboxylic acid was more reactive, together with (2) the formation of a more stable transitional 7-cycle conformation after the amine addition (see Supporting Information and Figure S7). ${ }^{34,35}$ 
Once all experimental conditions set up, it was possible to conjugate AuNPs to the series of SiNPs prepared with increasing initial APTES content and quantify the gold colloid density by TEM. Based on an estimation of the amount of gold colloids required to saturate the SiNP surface (see below), a AuNP:SiNP ratio of $10^{5}: 1$ was selected to perform the grafting reaction. Even after repeated washings, some remaining aggregates were still evidenced (Figure S7). Whereas they prevent direct measurement of Au content by XPS, they did not interfere with the determination of AuNP density on SiNP surface by TEM imaging (Figure 3). No AuNP were observed on the surface of unmodified SiNPs (Figure 3.1). For the lowest APTES concentration (sample 2 and 3), isolated dark colloids could be distinguished (Figure 3.2-3.3, arrows). A marked increase of AuNP density could be evidenced from sample $\mathbf{4}$, and the number of observable colloids seemed to progressively increase from sample 5 to 9 (Figure 3.4-3.9). Sample 10 had a distinct profile as its surface was coated with much larger particles (Figure 3.10).

To quantify gold nanoparticle density, the number of AuNPs was counted on a crown of 20 nanometers (Figure 4A) and the results gathered in Figure 4B. Note that due to the logarithmic $\mathrm{X}$ scale, sample 1 is not shown. Three different regimes can be distinguished. At low APTES content (samples $\mathbf{2}$ and 3), the AuNP number does not significantly vary and remains below 0.01 particles per $\mathrm{nm}^{2}$. At intermediate content, 0.01-0.1 APTES:SiOH ratios (samples 4 to 7), AuNP density increases up to $c a$. 0.08 particles per $\mathrm{nm}^{2}$ in an apparent linear manner. Finally, beyond an initial APTES:SiOH ratio of 0.1 (samples 8 to 10 ), a plateau is reached. Standard deviations were the most important in this last region so that no significant difference in AuNP density could be ascertained for the last four samples. 

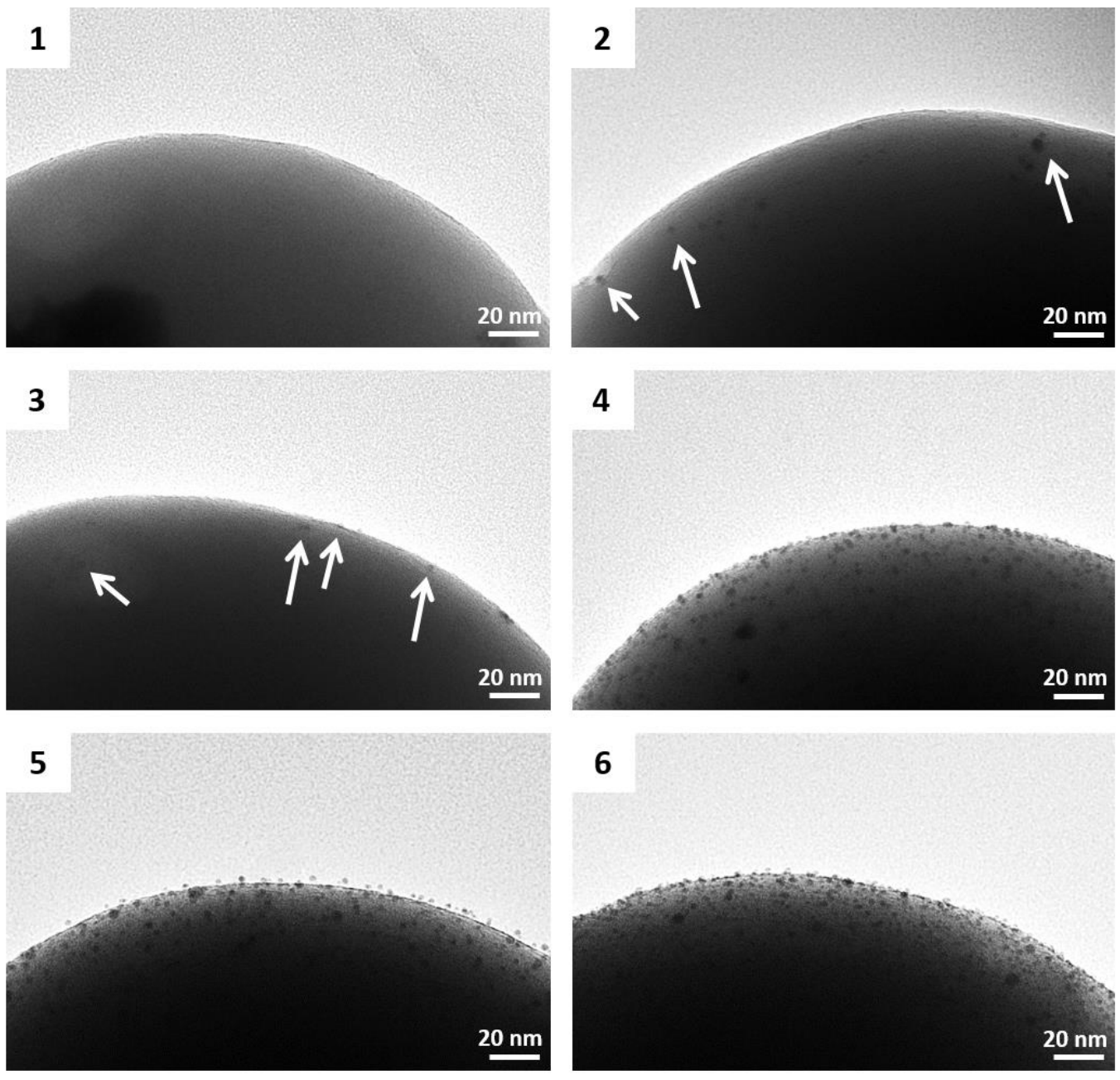

6
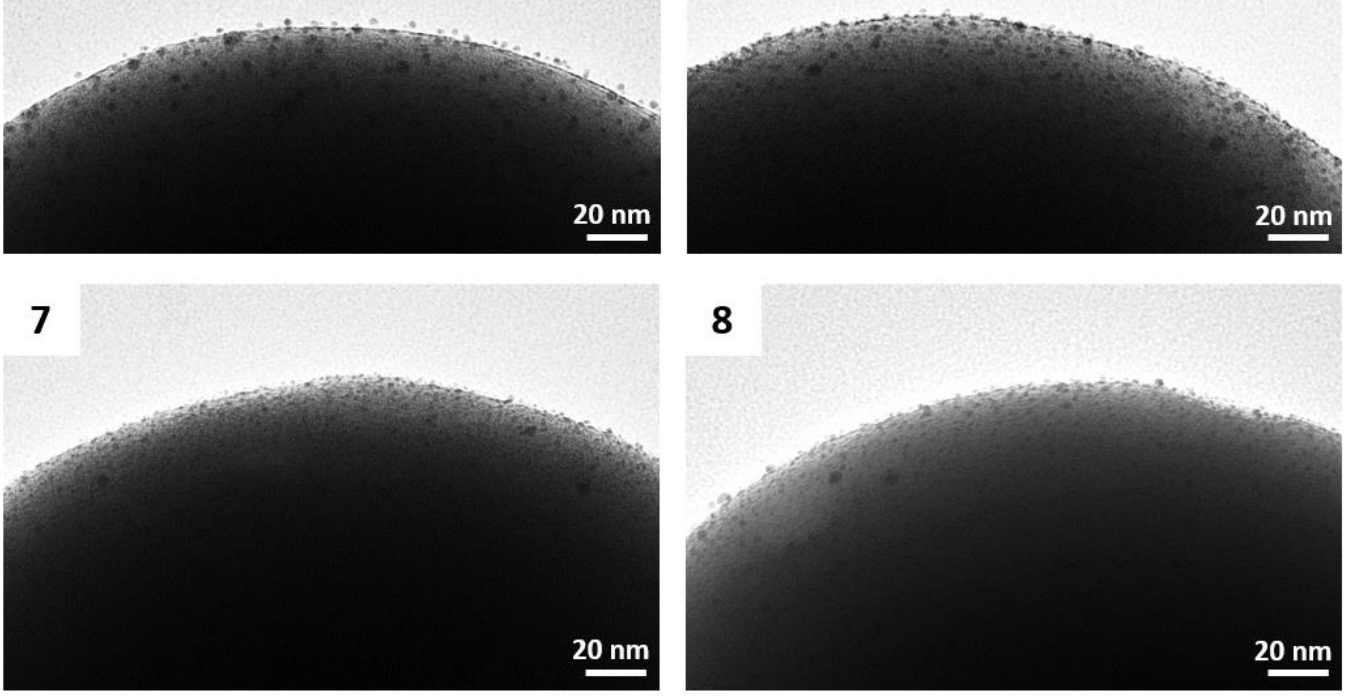

\section{9}

10
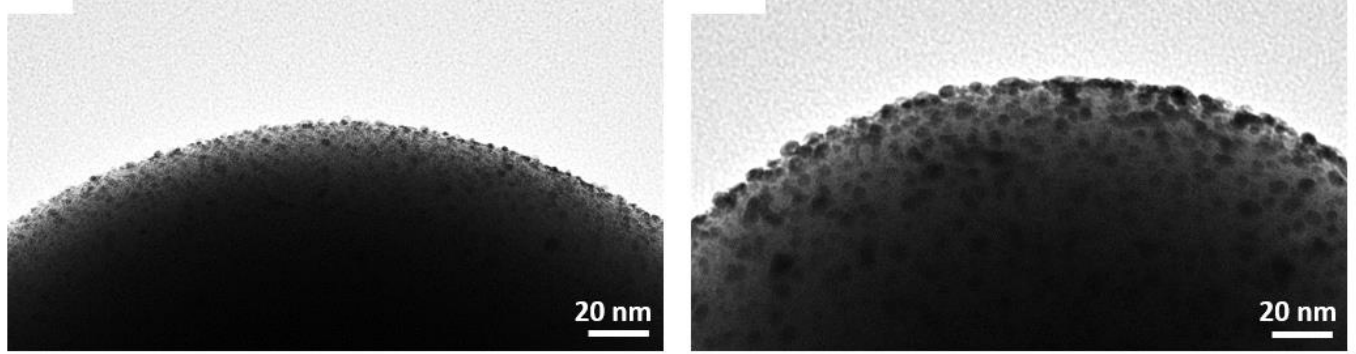

Figure 3. TEM images of APTES-modified SiNPs after AuNP conjugation. 
A

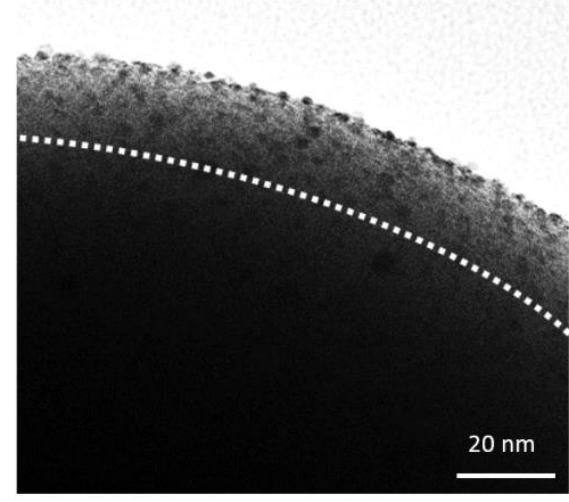

B

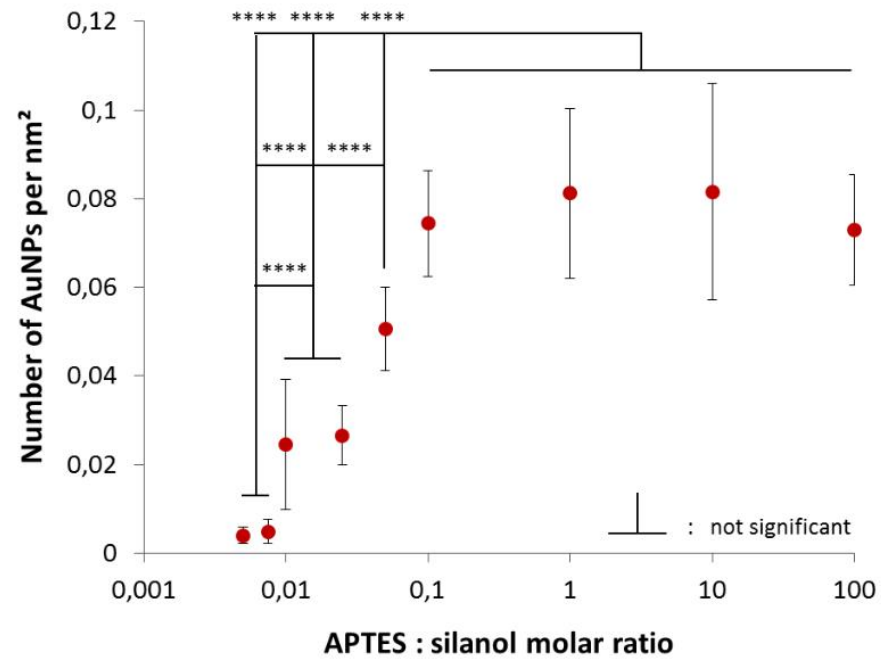

Figure 4. Evolution of the number of AuNPs per $\mathrm{nm}^{2}$ as a function of APTES:silanol stoichiometry. Each point represents mean \pm standard error of mean $(\mathrm{SD})(* * * * \mathrm{p}<0.0001$; calculated using ANOVA parametric test; 40 measurements on $10 \mathrm{SiNPs}$ per condition).

\section{DISCUSSION}

With the aim of preparing silica nanoparticles exhibiting a wide range of surface amine group density, bare SiNPs obtained by the Stöber process were reacted with APTES in conditions ranging from large surface silanol excess to large aminosilane excess. XPS allowed to ascertain grafting for initial ratio of 0.01 and above, i.e. when the amount of reacted APTES was significantly larger than the organic contamination of the sample. Moreover, the N/Si ratio increased almost linearly with initial APTES amount except for the highest tested ratio (i.e. 100), which suggests the saturation of SiNP surface (Figure 5A). 

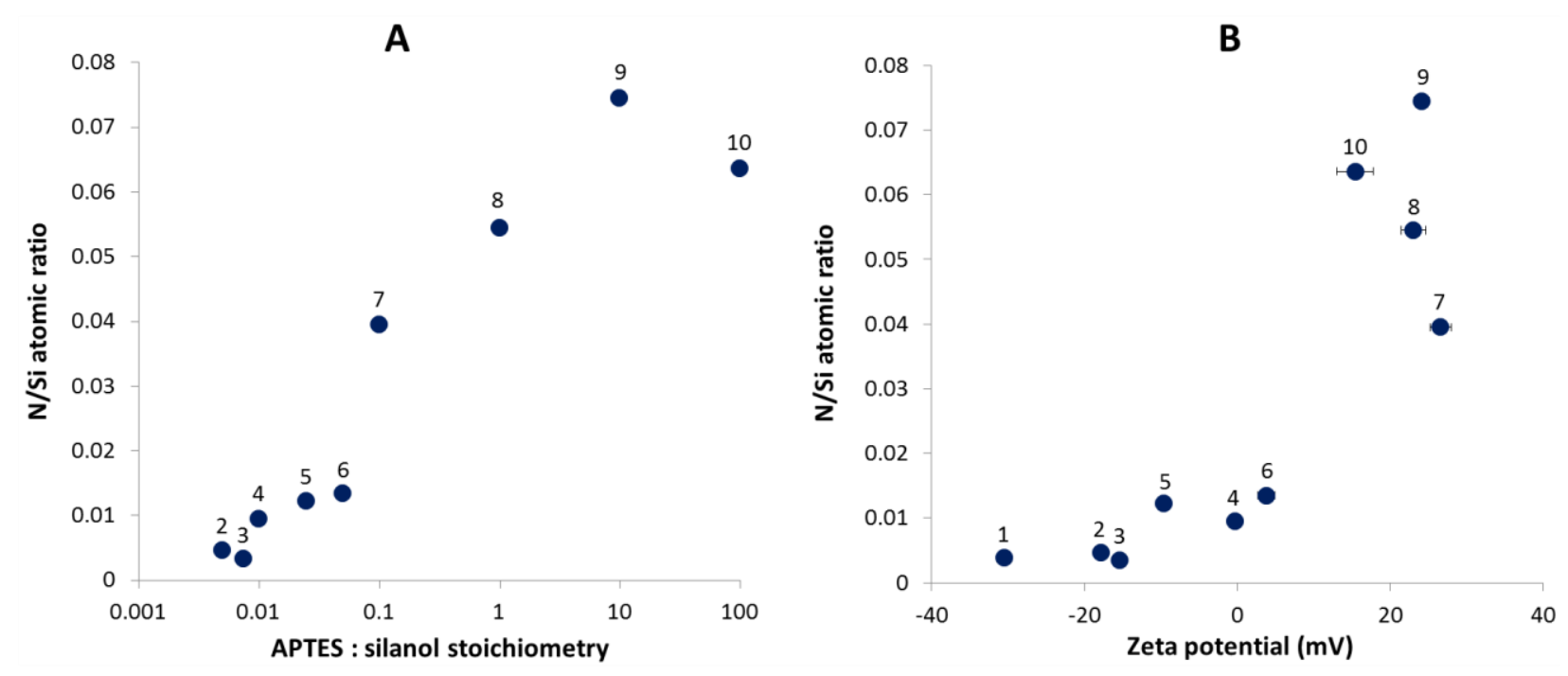

Figure 5. Evolution of the N/Si atomic ratio with (A) APTES:silanol stoichiometry and (B) measured zeta potential at pH 9.

Zeta potentials were also sensitive to the initial APTES concentration but with a strong dependence on $\mathrm{pH}$ (Figure 1). The measured zeta potentials result from a balance between silanol/silanolate groups $(\mathrm{pKa} \sim 3)$ and ammonium/amine groups $(\mathrm{pKa} \sim 10)$. Up to $\mathrm{pH} \sim 3$, combination of $\mathrm{NH}_{3}{ }^{+}$and $\mathrm{Si}-\mathrm{OH}$ makes the surface positive so that the measured zeta value at this $\mathrm{pH}$ is a good indication of the amount of grafted APTES. At $\mathrm{pH} \sim 9$, the surface charge results from $\mathrm{Si}_{-} \mathrm{O}^{-}$groups and fraction of the amine groups that are still in the $\mathrm{NH}_{3}{ }^{+}$form. Noticeably, grafting of APTES not only introduces amine groups but also decreases the number of $\mathrm{Si}-\mathrm{OH} / \mathrm{Si}^{-} \mathrm{O}^{-}$due to the formation of $\mathrm{Si}-\mathrm{O}-\mathrm{Si}$ (APTES) siloxane bonds. Thus the relationship between zeta potential and grafting extent is less straightforward. As a matter of fact, when plotting N/Si ratio as a function of zeta potential at $\mathrm{pH} 9$ (Figure 5B), one can notice that samples with close N/Si ratio can have very distinct $\zeta$ values (see samples 4 to 6) while samples with very close $\zeta$ values can have very distinct N/Si values (see samples 7 to 9). In parallel, XPS shows significant and non-monotonous variation of the $\mathrm{NH}_{2} / \mathrm{NH}_{3}{ }^{+}$ratio with initial APTES. This can explain why samples with low APTES density 
but high amine protonation rate can show close $\zeta$ values to those with higher silane content but lower ammonium relative amount.

The question is therefore raised about the origin of such variation in the $\mathrm{NH}_{2} / \mathrm{NH}_{3}{ }^{+}$ratio. It is in fact known that the protonation state of amine groups of grafted APTES strongly depends on its density and conformation on the silica surface. ${ }^{36}$ In particular acid-base reaction can occur with surface silanols and strong hydrogen bonds can also be established between close amines and ammonium groups. Such interactions, and their evolution with APTES content, are evidenced by the observed shift towards lower binding energies of the two XPS N1s peaks from conditions 1 to 10. Such shift can be associated with the decrease of the electronegativity of the elements surrounding the nitrogen atom where oxygen atoms of surface silanols are progressively substituted by nitrogen atoms of co-grafted APTES.

Altogether XPS and zeta potential measurements allow for evaluating the amount of grafted APTES but do not provide direct information on the spatial organization of the amine groups. To obtain such information, we have developed a strategy to achieve a robust and specific conjugation of electron-dense colloids to be observed by TEM.

To this aim, carboxylate-modified AuNPs with a diameter of $5.0 \pm 0.7 \mathrm{~nm}$ were synthesized to cope with both the surface conjugation requirement for specific interactions with amines, and size constraints for reaching acceptable resolution. As shown on Figure 4, a three-domain plot could be obtained with an initial low variation, an intermediate fast pseudo-linear increase and a plateau. To analyze these data further, the variations of AuNP density were plotted as a function of the N/Si atomic ratio obtained from XPS (Figure 6A). A similar curve was obtained, which incorporates the data corresponding to the bare SiNP (sample 1), allowing to define the limit of 
detection of our approach to $\mathrm{N} / \mathrm{Si}=0.01$. It also allows to perform a linear regression of "a" slope between samples 1 and $\mathbf{6}$ (Figure 6B). After converting the N/Si ratio as obtained from XPS into amine density (Table 1), it is possible to estimate that, in the linear region, the amount of amine groups per gold nanoparticles is $c a$. 30. When modeling the area of interaction between AuNP and SiNP surface by a disk of $5 \mathrm{~nm}$ in diameter, and assuming a density of $4.5 \mathrm{SiOH} . \mathrm{nm}^{-2}$, such a value corresponds to a situation where 1/3 of the surface groups are APTES molecules instead of silanols (Figure 6C). Altogether, in this region, the determination of the density of AuNPs allows for a quantitative determination of the density of amine groups on the SiNP surface.

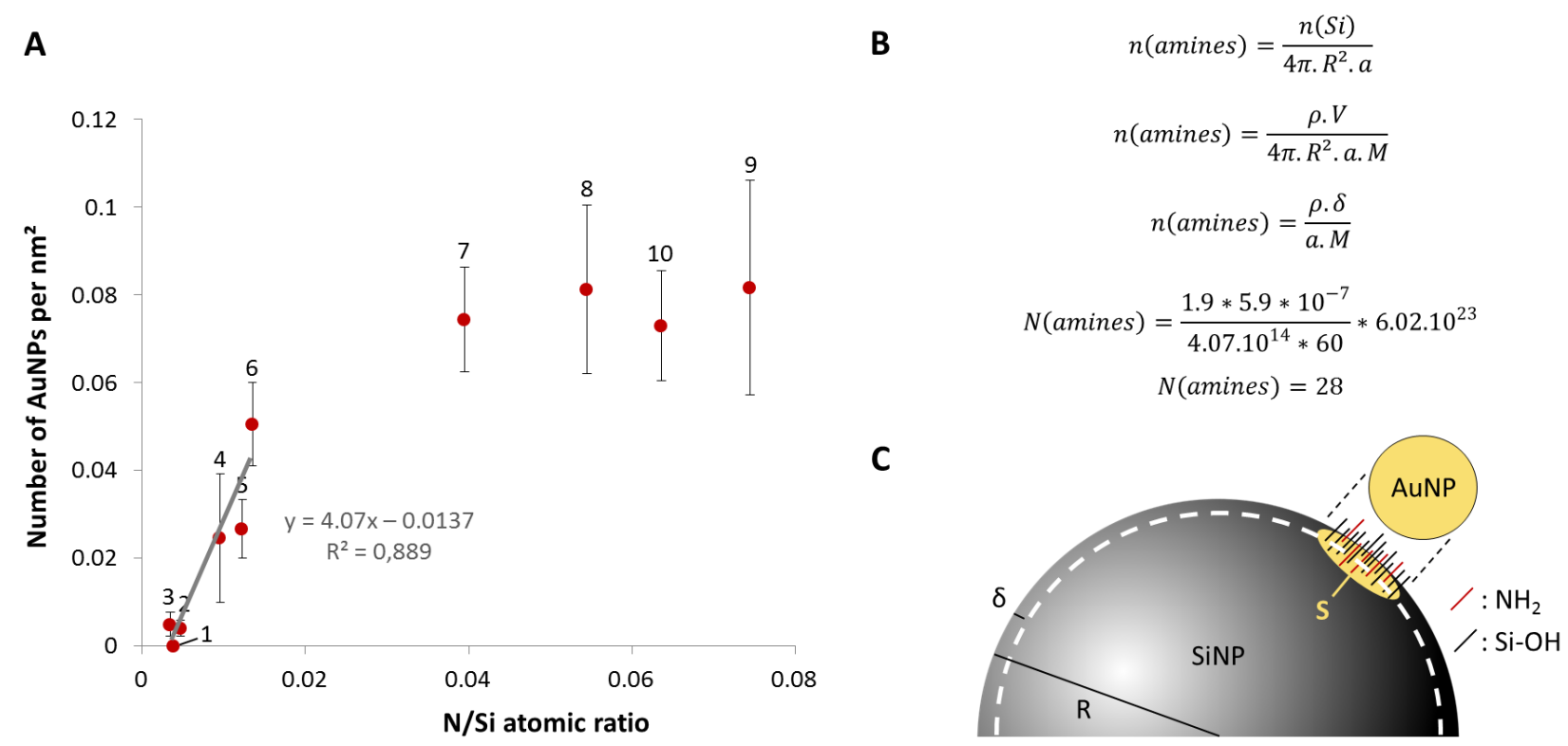

Figure 6. (A) Evolution of the number of AuNPs per $\mathrm{nm}^{2}$ as a function of N/Si atomic ratio. (B) Calculation of the number of amines necessary to bind one AuNP. (C) Scheme of a model of interaction between a SiNP of radius R, presenting 1/3 surface groups covered with APTES and a $5 \mathrm{~nm}$ AuNP.

In the last domain (samples 7 to 10) the measured density of AuNPs does not vary although the N/Si ratio continues to increase with initial APTES content, except for sample 10. Two reasons can explain such observation: either the SiNP surface is saturated from a steric perspective (i.e. 
AuNPs are at the highest possible packing density from sample 7) or APTES molecules are not grafted as a single layer. To examine the first hypothesis, one should consider that the surface of a silica particle is $c a .150,000 \mathrm{~nm}^{2}$ while that of a disk-shape equivalent to a gold nanoparticle is $c a$. $20 \mathrm{~nm}^{2}$. Therefore, the maximum AuNP density on an equivalent flat silica surface would be ca. 0.05 particles per $\mathrm{nm}^{2}$. Noticeably, this number is overestimated as the maximum packing of spheres is $c a .85 \%$ in 2D, assuming a random packing. Thus, surface saturation by AuNPs may be reached in the conditions of sample 6. The second possibility, that does not contradict the first one, is supported by the estimated amine density for samples $\mathbf{7}$ to $\mathbf{1 0}$ (Table 1) that is greater than the reported average $\mathrm{SiOH}$ density $\left(4.5 \mathrm{SiOH} . \mathrm{nm}^{2}\right)$ on the particle surface. This raises the question of the mechanisms of APTES grafting.

First, we can hypothesize a heterogeneous grafting. Indeed, it has been shown in the literature that APTES molecules can graft as clusters, thus defining functional domains (red spots in Figure 7). ${ }^{37}$ At low APTES density, there should be small randomly isolated domains, not large enough for the conjugation of carboxylate-modified AuNPs. This modelling matches well with the initial constant regime. The following linear regime can be explained by two potential evolutions: (1) the broadening of these initial domains (Figure 7A) or (2) the multiplication of domains with increasing APTES concentration (Figure 7B). When examining TEM images of samples 2 and $\mathbf{3}$, dispersed AuNP aggregates were more frequently observed than isolated single colloids. This would indicate local high density of amines. If these domains had further grown in size, then aggregates of increasing size should be observed with increasing APTES concentration. In contrast from sample $\mathbf{4}$ and above, such aggregates are no longer evidenced and isolated AuNPs appear homogeneously distributed on the surface, with an increasing density with N/Si ratio. This observation is therefore more in favor of the multiplication of amine domains of critical density. 

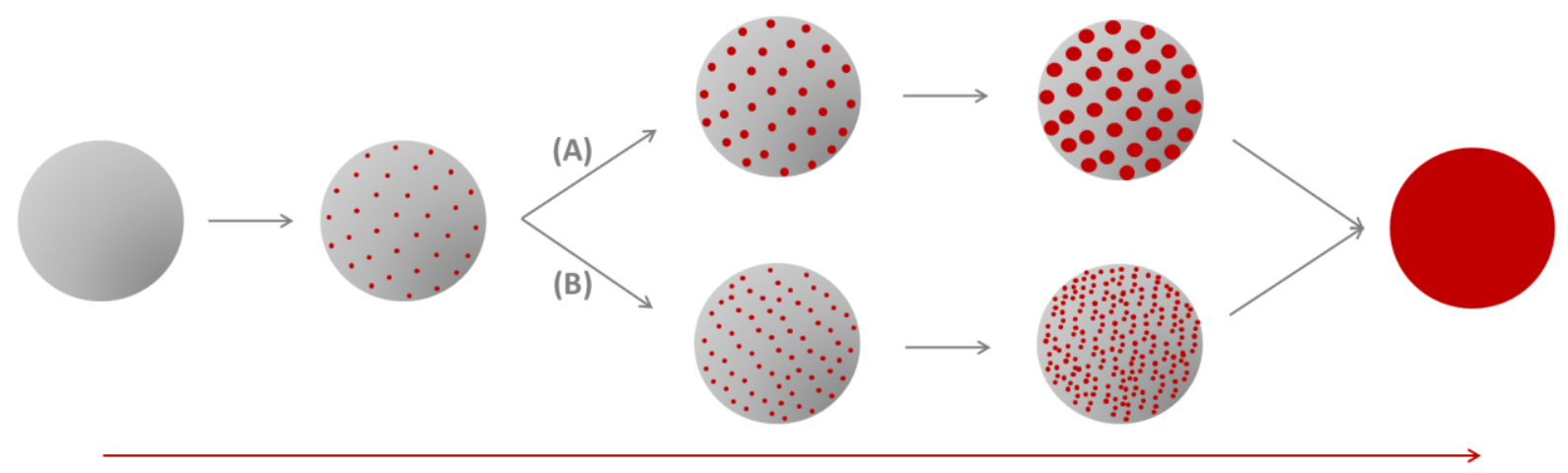

[APTES]

Figure 7. Schematic representation of APTES grafting via a heterogeneous model, as a function of APTES initial concentration in the reaction mixture. (A) Broadening of domains or (B) multiplication of small domains up to fullycovered SiNP-NH .

However, an alternative homogenous grafting mechanism needs to be considered (Figure 8). In that case, at least two regimes can be expected: if the average amine density is below the critical threshold, no AuNP should be grafted. Above this threshold, all particles should be able to bind the surface simultaneously. This model should result in two constant regimes, whether the SiNP is respectively not and fully covered with AuNPs (black curve). To refine this model, it is possible to add a cumulative distribution function, associating the probability for one AuNP to get grafted at $\mathrm{SiNP}-\mathrm{NH}_{2}$ surface with APTES concentration. Such a function usually corresponds to a sigmoid function $f$ of parameter $c$, which could match the experimentally-observed three-regime variation (red curve). 


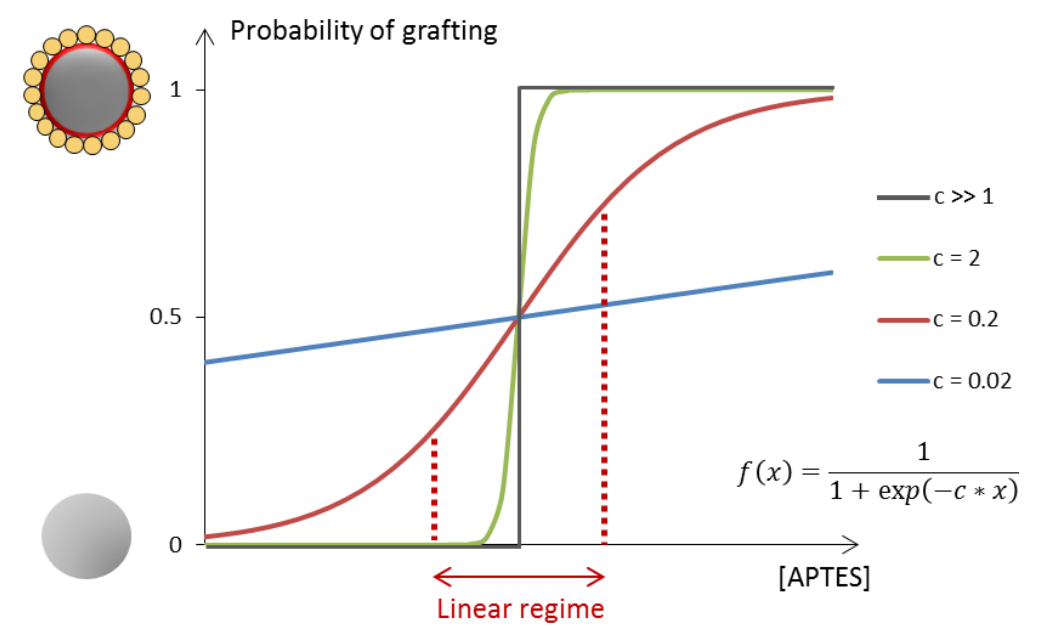

Figure 8. Schematic representation of potential regimes for APTES grafting via a homogeneous model. A cumulative distribution function $f$ is represented by a sigmoid of parameter $c$, which could explain the existence of an apparent linear regime.

In the present situation, parameter $c$ scales as the probability for one AuNP to contact with and covalently bind the SiNP surface. Thus the highest the conjugation efficiency, the steeper the slope (see green and blue curves). As already mentioned, preliminary attempts have been made using the HOBt/HBTU coupling system, known as less efficient than HATU (see Supporting Information and Figure S6 and S7). This would correspond to a lower $c$ value, for which intermediate coating density could be expected. This is consistent with our observations, where SiNPs were partially coated and with a variable density after coupling with HOBt/HBTU (Figure S6).

However, the homogeneous model would lead to the absence of AuNP grafting at low APTES concentration and cannot therefore explain the presence of AuNP aggregates observed for samples $\mathbf{2}$ and $\mathbf{3}$ that are clearly distinct from bare SiNPs (sample 1). Altogether a heterogeneous deposition of APTES with an increasing number of amine domains above the critical size appears as the most likely explanation. 
A final point to address is the structure of the APTES coating at high concentration (samples 7-10). As mentioned above, in these conditions, the estimated density of amines was larger than the reported average density of silanols on the surface of SiNPs. Moreover, XPS data showed that, whereas the relative amount of $\mathrm{NH}_{2}$ vs $\mathrm{NH}_{3}{ }^{+}$groups increased with APTES concentration for samples 2 to 6, it decreased for higher grafting density. This suggests that APTES grafting deviates from the formation of a monolayer covering the SiNP surface. In the literature, the presence of $\mathrm{NH}_{2}$ groups at $\mathrm{pH}$ well-below the $\mathrm{pKa}$ of primary amines is attributed to an acid-base reaction between ammonium and silanolates leading to amines and silanols. Therefore the observed decrease of neutral amines would suggest either that there is no available silanolates or that extra APTES molecules are not in close contact with the silica surface. These two possible, and not excluding, situations can be explained by considering that highly concentrated APTES preferentially prepolymerize into clusters before undergoing grafting on SiNPs. This completes the global picture of our model, with APTES grafting as clusters at the surface of SiNPs and resulting in potential APTES multilayers.

\section{CONCLUSION}

This work aimed at exploring a strategy allowing for the specific titration and localization of functional groups on the surface of nanoparticles. The use of $5 \mathrm{~nm}$ gold colloids bearing carboxylate functions and grafted on aminosilane-modified silica nanoparticles via a peptide coupling reaction allowed us to establish a correlation between amine density, as obtained from XPS, and gold colloid density, as obtained by TEM. Limitations of this approach were in the low amine density domain $\left(<1 \mathrm{NH}_{2} \cdot \mathrm{nm}^{2}\right)$, mainly due to the intrinsic limitations of routine XPS analysis, and the high amine density regime $\left(>5 \mathrm{NH}_{2} \cdot \mathrm{nm}^{2}\right)$ due to both the formation of multilayered APTES coatings and the full coverage of the silica surface by gold colloids. 
Our result also suggested that a minimal density of amine groups that can be calculated from the XPS-TEM correlation, was required to establish a stable interaction with gold colloids. While this contributes to the understanding of the mechanisms associated with APTES grafting on silica nanoparticles, it offered us the possibility to develop a strategy to probe and characterize ligand domains on the surface of patchy particles. Given the primordial importance of interfaces in colloid science, this strategy will be of great interest for the mapping of functional groups on the surface of nanomaterials and all the more suitable for the characterization of the interdistances of biological ligands on colloids interfacing with living systems.

\section{REFERENCES}

1. Wu, J.; Silvent, J.; Coradin, T.; Aimé, C. Biochemical Investigation of the Formation of Three-Dimensional Networks from DNA-Grafted Large Silica Particles. Langmuir 2012, 28 (4), $2156-2165$.

2. Laramy, C. R., O’Brien, M. N. and Mirkin, C. A. Crystal engineering with DNA. Nature Reviews Materials 20194 (3), 201-224.

3. Aimé, C.; Mosser, G.; Pembouong, G.; Bouteiller, L.; Coradin, T. Controlling the Nanobio Interface to Build Collagen-silica Self-Assembled Networks. Nanoscale 2012, 4 (22), 7127 7134.

4. Mout, R.; Ray, M.; Tay, T.; Sasaki, K.; Yesilbag Tonga, G.; Rotello, V. M. General Strategy for Direct Cytosolic Protein Delivery via Protein-Nanoparticle Co-Engineering. ACS Nano 2017, $11(6), 6416-6421$.

5. Banani, S. F.; Lee, H. O.; Hyman, A. A.; Rosen, M. K. Biomolecular Condensates: Organizers of Cellular Biochemistry. Nature Reviews Molecular Cell Biology 2017, 18 (5), 285298. 
6. Zhao, Y.-P.; Ye, D.-X.; Wang, G.-C.; Lu, T.-M. Novel Nano-Column and Nano-Flower Arrays by Glancing Angle Deposition. Nano Letters 2002, 2 (4), 351-354.

7. Snyder, C. E.; Yake, A. M.; Feick, J. D.; Velegol, D. Nanoscale Functionalization and SiteSpecific Assembly of Colloids by Particle Lithography. Langmuir 2005, 21 (11), 4813-4815.

8. Walther, A. and Muller, A. H. Janus particles: synthesis, self-assembly, physical properties, and applications. Chemical reviews 2013, 113 (7), 5194-5261.

9. López, V., Villegas, M. R., Rodríguez, V., Villaverde, G., Lozano, D., Baeza, A. and ValletRegí, M. Janus mesoporous silica nanoparticles for dual targeting of tumor cells and mitochondria. ACS applied materials \& interfaces 2017, 9 (32), 26697-26706.

10. Binks, B. P. Particles as Surfactants - Similarities and Differences. Interface Science 2002, $7,21-41$.

11. Haney, B., Chen, D., Cai, L. H., Weitz, D. and Ramakrishnan, S. Millimeter-Size Pickering Emulsions Stabilized with Janus Microparticles. Langmuir 2019, 35 (13), 4693-4701.

12. Manoharan, V. N.; Elsesser, M. T.; Pine, D. J. Dense Packing and Symmetry in Small Clusters of Microspheres. Science 2003, 301 (5632), 483-487.

13. Wang, Y.; Wang, Y.; Breed, D. R.; Manoharan, V. N.; Feng, L.; Hollingsworth, A. D.; Weck, M.; Pine, D. J. Colloids with Valence and Specific Directional Bonding. Nature 2012, 491 (7422), 51-55.

14. Xu, S.; Nie, Z.; Seo, M.; Lewis, P.; Kumacheva, E.; Stone, H. A.; Garstecki, P.; Weibel, D. B.; Gitlin, I.; Whitesides, G. M. Generation of Monodisperse Particles by Using Microfluidics: Control over Size, Shape, and Composition. Angewandte Chemie International Edition 2005, 44 (5), 724-728. 
15. Misra, A. C., Park, T. H., Carney, R. P., Rusciano, G., Stellacci, F. and Lahann, J. Selective Localization of Hierarchically Assembled Particles to Plasma Membranes of Living Cells. Small Methods 2019, 3 (5), 1800408.

16. Désert, A.; Hubert, C.; Fu, Z.; Moulet, L.; Majimel, J.; Barboteau, P.; Thill, A.; Lansalot, M.; Bourgeat-Lami, E.; Duguet, E.; et al. Synthesis and Site-Specific Functionalization of Tetravalent, Hexavalent, and Dodecavalent Silica Particles. Angewandte Chemie International Edition 2013, 52 (42), 11068-11072.

17. Chomette, C.; Duguet, E.; Mornet, S.; Yammine, E.; Manoharan, V. N.; Schade, N. B.; Hubert, C.; Ravaine, S.; Perro, A.; Tréguer-Delapierre, M. Templated Growth of Gold Satellites on Dimpled Silica Cores. Faraday Discussions 2016, 191, 105-116.

18. Désert, A.; Chaduc, I.; Fouilloux, S.; Taveau, J.-C.; Lambert, O.; Lansalot, M.; BourgeatLami, E.; Thill, A.; Spalla, O.; Ravaine, S.; et al. High-Yield Preparation of Polystyrene/Silica Clusters of Controlled Morphology. Polymer Chemistry 2012, 3 (5), 1130-1132.

19. Costi, R., Saunders, A. E. and Banin, U. Colloidal hybrid nanostructures: a new type of functional materials. Angewandte Chemie International Edition 2010, 49 (29), 4878-4897.

20. Hamon, C.; Martini, C.; Even-Hernandez, P.; Boichard, B.; Voisin, H.; Largeau, L.; Gosse, C.; Coradin, T.; Aimé, C.; Marchi, V. An Aqueous One-Pot Route to Gold/Quantum Rod Heterostructured Nanoparticles Functionalized with DNA. Chemical Communications 2015, 51 (89), 16119-16122.

21. Khalavka, Y., Harms, S., Henkel, A., Strozyk, M., Ahijado-Guzmán, R. and Sönnichsen, C. Synthesis of Au-CdS@ CdSe Hybrid Nanoparticles with a Highly Reactive Gold Domain. Langmuir 2018, 34 (1), 187-190. 
22. DeVries, G. A.; Brunnbauer, M.; Hu, Y.; Jackson, A. M.; Long, B.; Neltner, B. T.; Uzun, O.; Wunsch, B. H.; Stellacci, F. Divalent Metal Nanoparticles. Science 2007, 315 (5810), 358361.

23. Modena, M. M., Rühle, B., Burg, T. P. and Wuttke, S. Nanoparticle characterization: What to measure? Advanced Materials 2019, 31 (32), 1901556.

24. Mourdikoudis, S., Pallares, R. M. and Thanh, N. T. Characterization techniques for nanoparticles: comparison and complementarity upon studying nanoparticle properties. Nanoscale 2018, 10 (27), 12871-12934.

25. Chen, Y. and Zhang, Y. Fluorescent quantification of amino groups on silica nanoparticle surfaces. Analytical and bioanalytical chemistry 2011, 399 (7), 2503-2509.

26. Ciccione, J., Jia, T., Coll, J. L., Parra, K., Amblard, M., Jebors, S., Martinez J., Mehdi, A. and Subra, G. Unambiguous and controlled one-pot synthesis of multifunctional silica nanoparticles. Chemistry of Materials 2016, 28 (3), 885-889.

27. Baer, D. R. Guide to making XPS measurements on nanoparticles. Journal of Vacuum Science \& Technology A: Vacuum, Surfaces, and Films 2020, 3 8(3), 031201.

28. Rades, S., Hodoroaba, V. D., Salge, T., Wirth, T., Lobera, M. P., Labrador, R. H., Natte, K., Behnke, T., Gross, T. and Unger, W. E. High-resolution imaging with SEM/T-SEM, EDX and SAM as a combined methodical approach for morphological and elemental analyses of single engineered nanoparticles. RSC advances 2014, 4 (91), 49577-49587

29. Sokolowska, K., Luan, Z., Hulkko, E., Rameshan, C., Barrabes, N., Apkarian, V. A. and Lahtinen, T. Chemically Selective Imaging of Individual Bonds Through Scanning Electron Energy-Loss Spectroscopy: Disulfide Bridges linking Gold Nanoclusters. The Journal of Physical Chemistry Letters 2020, 11 (3), 796-799. 
30. Wang, W. and Vaughn, M. W. Morphology and Amine Accessibility of (3 - Aminopropyl) Triethoxysilane Films on Glass Surfaces. Scanning: The Journal of Scanning Microscopies 2008, $30(2), 65-77$.

31. Chen, G., Perez-Garcia, R., Danglad-Flores, J. and Riegler, H. Capillary-Enhanced Immobilization of Nanoparticles. The journal of physical chemistry letters 2017, 8 (24), 60946098.

32. Dems, D., Freeman, R., Riker, K. D., Coradin, T, Stupp, S. I. and Aimé, C. Multivalent Clustering of Adhesion Ligands in Nanofiber-Nanoparticle Composites. Acta Biomaterialia 2021, 119, 303-311.

33. Chen, S.; Kimura, K. Synthesis and Characterization of Carboxylate-Modified Gold Nanoparticle Powders Dispersible in Water. Langmuir 1999, 15 (4), 1075-1082.

34. Carpino, L. A., Imazumi, H., Foxman, B. M., Vela, M. J., Henklein, P., El-Faham, A., Klose, J. and Bienert, M. Comparison of the effects of 5-and 6-HOAt on model peptide coupling reactions relative to the cases for the 4- and 7-isomers. Organic letters 2000, 2 (15), 2253-2256.

35. Garner, P., Şeşenoğlu, Ö. and Kaniskan, H. U. S-(2-Pyrimidinyl)-and S-(2-(4, 6dimethylpyrimidinyl))-1, 1, 3, 3-tetramethylthiouronium hexafluorophosphates: novel reagents for in situ peptide coupling. Tetrahedron letters 2006, 47 (4), 483-486.

36. Golub, A. A., Zubenko, A. and Zhmud, B. V. $\gamma$-APTES modified silica gels: the structure of the surface layer. J. Colloids and Surf. Sci. 1996, 179, 482-487.

37. Elmlund, D., Le, S. N. and Elmlund, H. High-resolution cryo-EM: the nuts and bolts. Current opinion in structural biology 2017, 46, 1-6.

\section{ACKNOWLEDGEMENTS}


We thank Nicolas Delsuc and Amandine Vincent, from the Laboratory of BioMolecules (UMR7203) for their help and fruitful discussions concerning the peptide coupling, Sylvain le Grill for his involvement at the very beginning of the project. We thank Antoine Miche, from the Laboratory of Reactivity of Surface (UMR7197) for XPS measurements. We acknowledge financial support from the French state funds managed by the ANR within the Investissements d'Avenir program under reference ANR-11-IDEX-0004-02, and more specifically within the framework of the Cluster of Excellence MATISSE led by Sorbonne Université. N.D. thanks ENSPSL University for his PhD grant. 